\title{
Homesickness: A Systematic Review of the Scientific Literature
}

\author{
Margaret Stroebe \\ Utrecht University and University of Groningen \\ Maaike Nauta \\ University of Groningen
}

\author{
Henk Schut \\ Utrecht University
}

\begin{abstract}
Large-scale migration in contemporary society underscores the need to learn about the complex experience of leaving home and relocating within or beyond one's own country. Separate consideration of various types of stressors associated with geographic moves is called for, because these are likely to be associated with different (but mutually exacerbating) correlates and consequences, perhaps leading to comorbidity. Homesickness (HS) is a frequent component of relocation, known to be related to certain adjustment difficulties and health problems, as well as clinical disorders. However, an up-to-date, comprehensive overview of scientific investigations of HS is lacking. Therefore, this article systematically reviews empirical research on HS, focusing on temporary sojourns from home. Fifty-five articles that conformed to adequate design and methodological criteria were identified in the literature. These studies reveal indicative patterns of findings relating to the prevalence, consequences, predictors, and prevention/treatment strategies for HS. Gaps in knowledge about phenomena and manifestations associated with HS are also discussed. Importantly, it is demonstrated that the inclusion of stressors in the new place when assessing HS limits understanding. We suggest theory-guided directions for future research.
\end{abstract}

Keywords: homesickness, grief, mental/physical health, separation, relocation

Supplemental materials: http://dx.doi.org/10.1037/gpr0000037.supp

The World Health Organization has published remarkable statistics pointing to the large numbers of migrants in the world today, highlighting associated problems:

There are an estimated 1 billion migrants in the world today of whom 214 million international migrants and 740 million internal migrants. . . . Migration flows comprise a wide range of populations, such as workers, refugees, students, undocumented migrants and others, with each different health determinants, needs and levels of vulnerability.

Source: http://www.who.int/hac/techguidance/health_of_migrants/en/

These statements underline the need to understand the phenomena and manifestations associated with leaving one's home and possibly homeland, either temporarily or permanently, and either voluntarily or in some forced manner. Clearly, there are multiple ways in which contributions can be made to this highly complex endeavor. In our view, one fruitful approach is to examine the

This article was published Online First April 27, 2015.

Margaret Stroebe, Department of Clinical \& Health Psychology, Utrecht University, and Department of Clinical Psychology \& Experimental Psychopathology, University of Groningen; Henk Schut, Department of Clinical \& Health Psychology, Utrecht University; Maaike Nauta, Department of Clinical Psychology \& Experimental Psychopathology, University of Groningen.

Correspondence concerning this article should be addressed to Margaret Stroebe, Department of Clinical \& Health Psychology, Utrecht University, Box 80140, 3508 TC Utrecht, The Netherlands. E-mail: M.S.Stroebe@UU.NL different types of stressors and experiences associated with geographic moves independently of each other, and in a fine-grained manner, because these are likely to be associated with diversebut possibly mutually exacerbating — correlates and consequences. Homesickness (HS) is a frequently occurring phenomenon accompanying relocation, known also to go hand-in-hand with trouble adapting to a new environment and with related mental and physical health problems (van Tilburg, Vingerhoets, \& van Heck, 1996). So one strategy - the one we adopt here-is to isolate the experience of HS and to examine its related phenomena and manifestations by reviewing the accumulated body of scientific literature on this topic. Some reviews have specialized on subpopulations such as children/adolescents (e.g., Thurber \& Sigman, 1998) or college students (e.g., Thurber \& Walton, 2012); here, we provide broad coverage (cf. the earlier review by van Tilburg et al., 1996), indicating the subgroups studied in particular investigations. We first define HS and examine how it has been assessed, before evaluating what is known so far about its prevalence and duration, psychological and physical health consequences, as well as risk factors and correlates, and ways to prevent and/or treat HS. Finally, theory-guided directions for future research on HS are outlined.

\section{Review of Empirical Studies of HS}

The scientific study of HS is a relatively young discipline. Questions and doubts about its nature and conceptualization remain (cf. van Tilburg \& Vingerhoets, 2005), affecting how the empirical literature is reviewed. Researchers regard it as a complex phenomenon with manifestations occurring at different levels, 
ranging from emotional (e.g., yearning, loneliness), cognitive (e.g., preoccupying thoughts of home and attachment figures), social (e.g., withdrawing from those in the new environment), to somatic (e.g., loss of weight; cf. van Tilburg et al., 1996). As the studies reviewed show, some persons are more prone to HS than others (people are typically able to assess whether they suffer from it), and its severity ranges from mildly to extremely debilitating; it can be anticipatory (sometimes leading to a decision not to leave home). It is different yet similar in some ways to other constructs. For instance, HS and separation anxiety share the central theme of "excessive anxiety concerning separation from home" (American Psychiatric Association, 2013). Separation anxiety disorder is described with a possible manifestation of becoming HS "to the point of misery when away from home" (American Psychiatric Association, 2013). However, there are also differences: Not all separation anxious children experience HS, and separation anxiety may also occur in the home environment when an attachment figure leaves or may leave. Thus, HS and separation anxiety seem overlapping but also have distinctive features. Relatedly, separating symptoms of HS from correlates and consequences is also no easy matter (but the distinctions are necessary if we are to assess HS's impact). There are, for example, different HS correlates-like anxiety and depression-but these could conceivably be considered as consequences of HS too (we return to this issue later on). Furthermore, the focus of HS may differ (some persons missing the familiar physical environment; others longing for family and friends), raising measurement issues (e.g., how much weight to assign to different HS components).

Given these difficulties, our reviewing strategy is based on a strict definition of HS (and detailed reporting of particular investigations, e.g., in terms of their identification of correlates and consequences). Various definitions have appeared in the scientific literature. These differ in scope, with some focusing on separation from home (referred to later as the home factor; e.g., Vingerhoets, 2005), while others include relocation phenomena (the new place factor), as well as missing the familiar place and persons (e.g., Fisher, Elder, \& Peacock, 1990), while still others include related outcome variables such as depression (e.g., Eurelings-Bontekoe, Brouwers, \& Verschuur, 2000). To provide conceptual clarity, Stroebe, Schut, and Nauta (in press) developed the Dual Process Model of HS (DPM-HS), viewing HS as primarily a separation phenomenon for independent consideration from relocation stressors. The DPM-HS postulates dual, regulatory processes, whereby separation ("home") phenomena of HS and relocation ("new place") difficulties may co-occur and possibly be mutually exacerbating. Put simply, the difference between this approach and the major, preexisting one of Fisher (e.g., 1989; Fisher, Murray, \& Frazer, 1985) is that the latter includes multicausal dimensions, incorporating predispositional traits and adjustment to the new environment in definition and explanation of HS. By contrast, in line with the DPM-HS, Stroebe et al. (in press) suggested a new definition of HS as a "minigrief": "a negative emotional state primarily due to separation from home and attachment persons, characterized by longing for and preoccupation with home, and often with difficulties adjusting to the new place." Adopting this narrower definition provides focus for assessing the empirical studies. The extent to which investigators have followed this narrow approach or a broader one such as Fisher's will become evident in the review.
The scientific studies of HS that form the basis for our review are presented online as supplemental material. These focus on prevalence and duration, as well as on the relationship between HS and health consequences. The scope of the investigation on home and new place factors is detailed. Included are quantitative studies that have investigated HS, its correlates, risk factors, and outcomes among children and adolescents, as well as adults. We formulated the following research questions for the current review: (1) How has HS been operationalized and measured? (2) What is known about the prevalence and duration of HS? (3) Which correlates and consequences of HS have been identified (physical and psychological health; preventive and risk factors)? (4) Do studies investigate HS as an attachment to home, or also as an adjustment to the new environment phenomenon (the home-new place factors), and if so, do they find meaningful differences between the two? (5) What is known about prevention and intervention for HS?

Before examining the research studies to seek answers to these questions, we give background information about our research strategy and introduce the results of the literature search.

\section{Background Information}

\section{The Search Strategy}

Review of the empirical literature was based on extensive searches. The primary search was conducted using Scopus (last update: October 3, 2015). PsycINFO and Medline were searched for overlap and distinctions with Scopus (November 27, 2013; rechecked June 3, 2014). Other sources were checked as well (PubMed; Google Scholar; reference lists). The flow of information is shown in Figure 1. The main search word was homesick*; other checks were made for: relocation; separation; separation anxiety. In Scopus, the search term was Homesick*; in title, keywords, abstract; all types of documents, all time, English language; social sciences and humanities/health sciences.

Records were screened at the Abstract level for their relevance to the HS topic; a broad range of contributions was retained at this stage, including qualitative and case studies, for potential inclusion in the text (but not the empirical studies' review). Following this, all full text articles were assessed for eligibility in the empirical review. Reasons for exclusion were the following: (1) permanent move (e.g., immigration, emigration, refugees); (2) other topic (e.g., acculturation); (3) HS not focal to the investigation (e.g., HS included as one of many stressors); (4) not English language; (5) no full text available (where possible, after contacting first and/or second author); (6) no data, poor design/methodology; (7) qualitative investigation.

To elaborate: We followed the rationale outlined earlier, severely restricting the research scope to HS, even though the ultimate interest extends to relocation more generally. We covered limited-stay sojourns away, those where there is the prospect of returning home, but not long-term or permanent relocation (e.g., among the elderly into care facilities). The latter are likely not only to be associated with HS but also with other complicating economic, political, and/or social factors, ones that could complicate investigation of the HS phenomenon. Thus, studies of migration in general were also excluded (e.g., Green, 2011). For similar reasons, investigations of culture shock (e.g., Ward, Bochner, \& Furnham, 2001) are beyond the current scope. Although this 


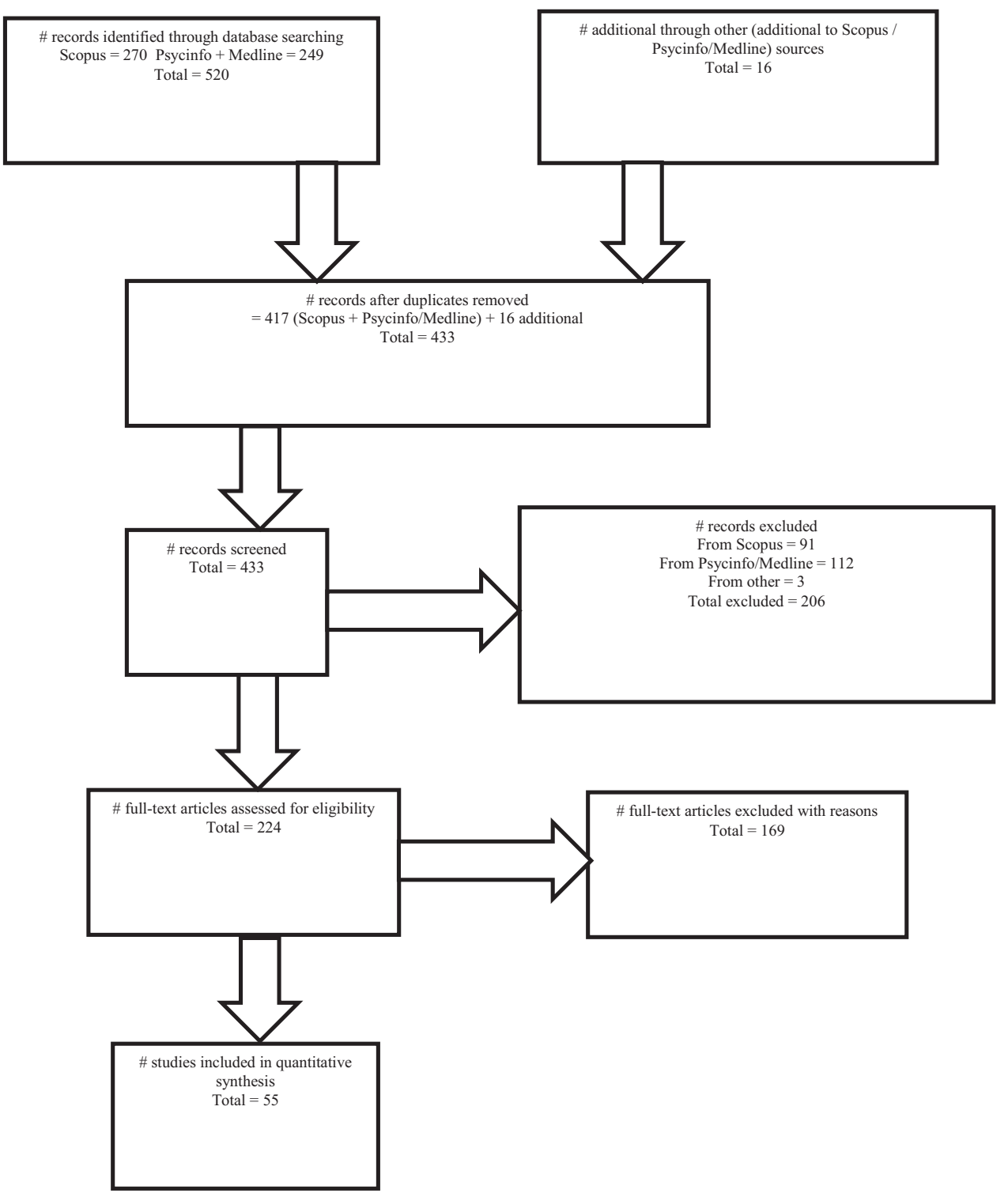

Figure 1. Flow of information through the different phases of the review.

phenomenon may be related to HS, it is distinct from it, because culture shock stems from confusion about the norms of the new culture and a sense of social difficulty (cf. Poyrazli \& Lopez, 2007). Also excluded were studies where the focus is general adjustment (e.g., to college) and not specifically HS, or where the focus is on sojourner adjustment (cf. Pedersen, Neighbors, Larimer, \& Lee, 2011). Finally, we excluded vacation effects on health and well-being (e.g., Nawijn, 2010), and acculturation (stress), where studies sometimes mention but do not centrally examine HS (e.g., Vergara, Smith, \& Keele, 2010; Yu et al., 2014).

Three further points about the in/exclusion criteria merit elaboration. First, a number of studies were excluded because they examined adjustment to a new environment in broader context, identifying HS only among factors contributing to difficulties (e.g., Hansson, Clausson, \& Janlöv, 2012). Second, one concession was made regarding methodological stringency: Some included studies relied on single-item HS measures. Given the paucity of information with psychometrically valid scales particularly among children, it was deemed better to retain these studies (particularly because a single question about HS is likely to be quite valid). Third, as evident in the online supplemental material, HS has mostly been examined as a dependent variable, while occasionally it is included as an independent variable, a stressor.

\section{Results}

We retrieved 55 studies on the topic of HS, including various subgroups that have been the focus of HS research (see online supplemental material). Many studies have examined HS in young children leaving home for schooling/recreation (boarding school in United Kingdom, e.g., Fisher and colleagues; residential summer camps in Canada and United States, e.g., Thurber and colleagues) 
Adolescents and young adult studies have been comprised mostly of student samples; that is, those who study in locations different from that of their families of origin, including universities and colleges (for a review, see Thurber \& Walton, 2012). Such samples also extend to specific student subgroups, such as nurses and international students of various disciplines, with some comparing HS among different nationalities (e.g., Poyrazli \& Lopez, 2007). Other samples have included hospitalized children (e.g., Thurber, Patterson, \& Mount, 2007). One study on HS was conducted among children with learning difficulties at a respite unit (Radcliffe \& Turk, 2008). With adult samples, Eurelings-Bontekoe and colleagues (e.g., Eurelings-Bontekoe, Duijsens, \& Verschuur, 1996a) have studied HS among military personnel, van Vliet (2001) expatriates and others have looked at HS among transferred employees (see Burt, 1993) or foreign employees (EurelingsBontekoe et al., 2000). Next, we address the questions posed earlier for our HS review: measurement issues, prevalence and duration, examination of correlates and consequences of HS, theoretical orientations; and finally, prevention and treatment.

\section{The Measurement of HS}

Examination of the studies shows that various indices, ranging from single questions to multiple-item scales, have been used to assess HS. Use of a scale is probably not necessary for the categorization of persons who are prone to be "homesick" versus "not homesick"- for which a single question/indicator may suffice, because respondents probably have little difficulty assigning themselves to one of these two categories. However, a scale taps nuances of the experience of HS. A variety of questionnaires has been constructed and used in the empirical studies. Researchers have adopted various strategies to develop these scales, at times basing their items on personal reports of HS experiences and reactions, or surveying expert opinions (see Vingerhoets, 2005). Some of the scales have been theoretically based, such as van Vliet's (2001), which was derived from stress and attachment theory, Fisher's (1989) relocation inventory, which stemmed from her sociological analysis of the HS situation, and Archer, Ireland, Amos, Board, and Currid's (1998) derivation from a grief/loss perspective.

The main HS questionnaires are summarized in Table 1, showing the range of HS dimensions included in each measure, reflecting researchers' theoretical approaches. We retrieved nine different self-report questionnaires; six were specific for adults, two for youth, and one seemed suitable across the life span. As can be seen, these self-report evaluations of HS cover a range of subjective feelings, cognitions, and behaviors. It is noteworthy that, although the scales differ in scope, most include items assessing adjustment to the new as well as aspects of missing the old environment, in line with the theoretical approach of Fisher (1989), outlined earlier. Archer et al. (1998), despite deriving their scale from the bereavement literature, reported the emergence of a second factor, namely dislike of the new environment. Although items were designed to parallel grief reactions (e.g., anger), they covered new locality aspects (e.g., "I hate this place"). Given the two factors, Archer et al. (1998) concluded that HS was not a unidimensional state. In general, examination of the empirical studies indicates that even when two (or more) such factors emerge, both are considered integral to HS; it is simply regarded as a multidimensional phenomenon. Furthermore, while some investigators include subscale analyses (e.g., Archer et al., 1998; Burt, 1993; Flett, Endler, \& Besser, 2009; Stroebe, van Vliet, Hewstone, \& Willis, 2002), total scores on HS rather than attachment-to-home versus adjustment-to-new-place dimensions are generally the basis for analyses and conclusions. The two dimensions typically correlate quite highly (e.g., Beck, Taylor, \& Robbins, 2003), but there are also indications that they have differential correlates (Stroebe et al., in press), thus raising the question whether maladaptation to the new place should really be considered integral to HS.

In line with our own theoretical approach, we would recommend two possibilities regarding scale selection, for future research: (1) that a "Homesickness Questionnaire" exclusively focusses on home separation but that the research investigation also includes a questionnaire on new place adjustment; (2) if — as has frequently been done so far- "new place adjustment" items are included in the HS questionnaire, that the latter are analyzed and reported separately, rather than combining them with the home-focused items and suggesting that they add up to HS. This opens the possibility of using existing data sets to reanalyze the findings on home and new place stressors separately (avoiding psychopathological measures of general stress/anxiety/depression-again, these need separate measurement). A home-separation HS questionnaire can be selected or adapted from those listed in Table 1. Items for a new place adjustment questionnaire can likewise be derived from those that include specific new place items, and/or from questionnaires in the broader adjustment field (e.g., Baker \& Siryk, 1999; Zahra et al., 2014). For example, Zahra et al.’s (2014) "Work and Social Adjustment Scale" covers impairment in work, home management, social and private leisure activities, and close relationships, representing a variety of new place stressors.

There are some additional concerns about the HS questionnaires, which also speak for following the guidelines outlined previously. As mentioned earlier, they sometimes include other psychological symptoms, such as depression and loneliness, which potentially confounds the HS measure with outcome variables. If depression is included in the HS scale, and the impact of HS on psychological adjustment in terms of symptoms of depression is being assessed, the correlation between HS and the outcome measure of depression will be artificially inflated. However, extreme negative mood, for example, is integral to HS (one is distressed about separation from home) making it difficult to separate depression of HS symptoms from depression as a consequence of HS. A solution may lie in restricting the former to specific separation items (e.g., low mood because of yearning for home) from generic indices of depression (e.g., unspecific anhedonia). Furthermore, only some (e.g., Fisher; see Fisher et al., 1990) look at frequency in occurrence, as well as intensity of HS. While most limit the focus to presence-absence or intensity of HS symptoms, chronicity (persistence of HS; high intensity HS symptoms over time) has also only sometimes been investigated (e.g., van Tilburg, Vingerhoets, \& van Heck, 1999). Most authors report adequate psychometric properties of their scales although Thurber and Walton (2012), reviewing student studies, reported only 2 psychometrically sound measures, namely their own (Thurber \& Sigman, 1998) and Archer et al.'s (1998). As is also evident in Table 1, very few measures are suitable for children or adolescents. Also, reports from the perspective of parents, (temporary) carers, or teachers on 
Table 1

Homesickness: Measures

\begin{tabular}{|c|c|c|}
\hline Authors & Scale (for adult/child) & Dimensions (home [separation]/new place) \\
\hline $\begin{array}{l}\text { Archer et al. (1998); see } \\
\text { also Ireland \& Archer } \\
\quad(2000)\end{array}$ & $\begin{array}{l}\text { Homesickness Questionnaire (HQ), } \\
\text { 33-item measure Adult }\end{array}$ & $\begin{array}{l}\text { Home separation and new place. Items } \\
\text { covered cognitive preoccupation; home- } \\
\text { related distress; maintaining ties; home- } \\
\text { related dreams; restlessness, anger and } \\
\text { guilt; loss of self, identification, and } \\
\text { avoidance }\end{array}$ \\
\hline $\begin{array}{l}\text { Eurelings-Bontekoe et al. } \\
\text { (1994, 1995, 1996a, } \\
\text { 1996b, 1998, 2000). } \\
\text { See also Verschuur, } \\
\text { Eurelings-Bontekoem } \\
\text { \& Spinhoven (2001) }\end{array}$ & $\begin{array}{l}\text { 1. A HQ, 87-item scale; reduced to } \\
\text { 29-item scale. 2. 9-item } \\
\text { checklist. 3. Self-reported HS } \\
\text { (long-lasting; holidays only) } 4 \text {. } \\
\text { HS Vulnerability Questionnaire } \\
\text { (HVQ) final version by } \\
\text { Verschuur et al. (2001), see } \\
\text { below }\end{array}$ & $\begin{array}{l}\text { 1. Included biographic q's, personality, } \\
\text { coping, etc. Only } 1 \text { subscale = } \\
\text { retrospective homesickness (HS)-related. } 2 \text {. } \\
\text { 9-item checklist: Desire to go back; } \\
\text { depressed mood; loss of interest; change } \\
\text { appetite, weight; sleeplessness, energy loss } \\
\text { restless or slow; fatigue; concentration } \\
\text { problems. }\end{array}$ \\
\hline
\end{tabular}
Fisher (1989); Fisher \& Dundee Relocation Inventory Hood (1987); see also (DRI); adults, boarding school Smrcek \& Stiksrud samples. (1994)

Porritt \& Taylor (1981) Homesickness Symptoms Scale Adult

Shin \& Abell (1999)

The Homesickness \& Contentment Scale Adult

Smrcek \& Stiksrud (1994) Germany

Thurber \& Weisz (1997b) United States

Thurber \& Sigman (1998)

van Tilburg,

Vingerhoets, \& van Heck (1997)

Verschuur et al. (2001)

van Vliet (2001); van

Vliet et al. (1998)

Adapted Fisher's (1989) Dundee Relocation Inventory for Germany. Extra items on uni. life \& tasks, inc. studies. Postadolescent

Ways of Coping with child/adolescent (WOCH)

Rate Your Day-Revised (RYD-R) Scale Child/adolescent

Adult Homesickness Coping Questionnaire (AHCQ) Adult (HVQ) Adult The Utrecht Homesickness Scale (UHS) Adult
Home separation and new place. 1.

Preoccupation with home (family, friends, home objects, routines). 2. Attitudes to new environment (dislike of certain features, being lost, overwhelmed, lonely, afraid). 2nd revision (Fisher, 1989, p. 139) $=26$ items, 3-point scale.

18 items derived from writings about common grief reactions \& attachment behaviors. Plus 1 item "I was homesick".

20 items evolved from earlier 30-item HS, loneliness \& depression scale. HS subscale 5 pt Likert-type: want to go back; write emails home; feel HS; miss friends and family. Homesickness questionnaire. For

5 HS factors: missing; satisfaction; lonelinesscommitment; achievement and residential.

15 items (including open item), measuring frequency and effectiveness of HS coping.

15-item self-report measure of positive, negative emotions and HS.

4 factors: Social support, positive thinking/ distraction, turning to religion, mental escape.

See Eurelings-Bontekoe et al. studies.
20 items; 5-factor structure: missing family; loneliness; missing friends; adjustment difficulties; ruminations about home

\author{
Comments
}

Identified 2 factors: attachment to home; disliking university. Did some separate analyses of these factors. Ireland and Archer (2000) made the HQ applicable for young offenders.

A. Vulnerability to, but not a HSfocused measure. B. Only one item on HS (but it must be endorsed for HS), covers consequences, HS = depressive and melancholic complaints. 1 and 2 both selfconstructed, little validation, HS Vulnerability Questionnaire (HVQ) was validated. N.B. it covers variables, such as personality dimensions, that are predictive of HS, but clearly not specific HS measures.

Includes outcome variables, also in DRI -2nd version (e.g., general adaptation and satisfaction items) as well as home and new environment related ones, a potential confounder if HS's impact on mental health outcome is examined.

Not used (extensively?) by others?

Culturally sensitive to Asians and international students. Contentment subscale covers adaptation to current situation, including emotions.

The dimensional approach follows Fisher, i.e., integrating current situation factors; different/additional components.

Based partly on Spirito et al.'s KIDCOPE; interviews.

For children.

Restricted to women. Ways of coping related to diverse aspects of HS experience. But no data collected to examine which coping strategies are more effective than others.

Validation study of earlier-constructed scale.

Based on previous research (e.g., by Fisher; Eurelings-Bontekoe); 100 respondents defined HS, their answers supplemented the above. Designed for broader application than child- and student-specific HS scales. the child's HS seem to be rare, even though it is quite common in youth assessment to evaluate emotional difficulties from a multiinformant perspective.

While most of the scales included in Table 1 were designed to assess the experience of HS specifically, some were not. Some researchers have extended the scope to assessment of related concepts, or to different types of assessment. Eurelings-Bontekoe team's research produced a vulnerability to HS scale, not one of the HS reaction (e.g., Eurelings-Bontekoe, Tolsma, Verschuur, \& Vingerhoets, 1996b; Verschuur, Eurelings-Bontekoe, \& Spinhoven, 2001). Although a vulnerability to HS scale is potentially useful, there are a number of limitations regarding this particular 
scale and its construction. Perhaps most important, the validation study by Verschuur et al. (2001) was cross-sectional, so the identified variables (such as externalization of feelings) cannot be regarded as established predictors of HS. Van Tilburg, Vingerhoets, and van Heck (1997) constructed a coping with HS questionnaire, to look at (in)effectiveness of coping strategies. However, these investigators did not examine the efficacy of different coping strategies in dealing with HS, stressing that this still needed to be done. Thurber developed measures appropriate for children including a coping scale (Thurber \& Weisz, 1997b, the Ways of Coping with Homesickness questionnaire (WOCH), and the Rate Your Day Revised (RYD-R; Thurber \& Sigman, 1998).

Sometimes HS has been added as a subscale in more general adjustment measures (e.g., Sandhu \& Asrabadi's, 1994, acculturative stress scale). Fisher (1989; see her appendix) also designed a broader measure, The Dundee Relocation Inventory, which assesses general adaptation, as well as feelings of satisfaction, distress, disorientation, and preoccupation with home-related thoughts (see Vingerhoets, 2005, for critical evaluation). Finally, Shin and Abell (1999) produced a scale that is culturally sensitive for Asians, the Homesickness and Contentment Scale.

Turning from questionnaire measurement to physiological markers of HS: Promising new research in this domain is emerging. Steiner and Coan (2011) used frontal electroencepalogram (EEG) asymmetry to separate out affective experience from memory-based beliefs in students' self-reports of HS. Relatively greater right frontal EEG asymmetry was associated with greater feeling-based, experiential reports of current HS, whereas no associations were found between frontal EEG asymmetry and beliefbased, retrospective reports of their HS. Thus, for one thing, this cautions that retrospective reporting of HS may be qualitatively different from reports of current HS.

In conclusion: There is need for consensus among researchers regarding the measurement of HS (while remaining open to improvements), not least so that the results of empirical studies can be better compared. HS instruments should separately not only assess but also analyze missing the old and adaptation to the new environment. In our view, questionnaires need to focus on HS per se; that is, to exclude its correlates and consequences. Scales may include adjustment problems as a separate construct, but they should not be integrated in HS per se. Scales should not include other incidental constructs or outcome variables. For example, including such words as "depressed" or "anxious" in the assessment of HS can inflate estimates of the correlation between HS and negative emotional outcomes.

Clearly, there is room for finer-grained measurement; for example, it would be useful to plot HS curves over time, following the lead of Nawijn (2010) for holiday happiness, or to use modern digital equipment and software for monitoring HS across time in the naturalistic environment. Frequent measurements allow for examining temporal relations between relevant constructs (de Vries, 1992). For instance, one could investigate whether feelings of nonadjustment to the current environment precede feelings of HS, or vice versa. Regarding children and adolescents, it may also shed light on the role of parents during the separation: How does contact with the home environment affect current and later emotions in children? Finally, establishing physiological and biological markers of HS are likely to prove useful for scientific understanding of HS.

\section{Prevalence and Duration of Homesickness}

It is difficult to make firm statements about prevalence and duration of HS, not only because of the measurement difficulties mentioned previously, but also because of the complex nature of the HS phenomenon (e.g., home-new place factors; different HS trajectories), subgroup differences (samples cover a variety of subgroups for whom prevalence varies), and because-where prevalence is concerned-HS has been assessed at different points in time after leaving home (van Vliet, 2001). It remains unclear too, when reactions to missing home should be called "homesickness" - what threshold should one adopt? Nevertheless, some general indications can be derived from the empirical studies.

We retrieved 14 studies on prevalence and duration of HS, with much variability in samples. Although the differences in prevalences across studies are clearly visible (see also the online supplemental material), it can be said that rates are generally far from negligible, with HS reported in quite large numbers of those who leave home. To illustrate, van Vliet (2001) provided some useful comparisons from his 3 projects (cross-sectional and longitudinal studies of students, and a longitudinal study of expatriates). While the student studies' prevalence estimates were as high as $48.7 \%$ and $45.6 \%$, a lower rate- under $40 \%$ - of expatriates experienced HS after relocation. Respondents (expats and controls) who were still at home at the first measurement point, were all asked whether they had felt HS in the past 4 weeks. So for the expats, this could have reflected anticipatory HS. It is interesting that before leaving, only $13.7 \%$ of the expats said that they already felt HS (and this \% included rarely, as well as sometimes, often, or very often). This was even lower than the rate for a control group, comprised of acquaintances of the expats, who were similar to them but who did not intent to leave home. $20.8 \%$ of these controls reported HSperhaps suggesting a HS self-selection process (more expats also self-reported to be securely attached than controls). Although the expats HS levels increased on relocation, the controls' levels remained roughly stable at around $20.0 \%$ (i.e., when asked about HS during the past 4 weeks).

Prevalence rates vary within student populations. Using the same Utrecht Homesickness Scale, Stroebe et al. (2002) found higher percentages of homesick students in the United Kingdom $(80 \%)$ than in the Netherlands $(50 \%)$, perhaps because of differences in accessibility to home. Vastly different percentages have been reported for other countries too, with Carden and Feicht (1991) finding that $19 \%$ of American and $77 \%$ of Turkish students attending universities in their own countries could be classified as homesick in their first year (based on a cutoff point of $1 S D$ above the mean group rating on a HS questionnaire). Prevalence information is also available for other subgroups, for example, Eurelings-Bontekoe et al. (2000) found serious HS among $18.9 \%$ of foreign employees (including depressive and melancholic complaints) while $30 \%$ had less severe HS.

What about prevalence of HS among children and adolescents? Again, different ways of assessing HS account for differences in estimates. On the one hand, Thurber (2005) found that as many as $94 \%$ of boys at summer camp (99\% of first-year boys) reported some HS on at least 1 day of their 2-week stay (Thurber, 2005). On the other hand, he found that many fewer, $18 \%$, had moderate to high HS levels with 7\% also reporting severe depression and anxious symptoms (Thurber, 1999; Thurber \& Walton, 2012). 
Prevalence is also likely to vary according to the specific separation from home experience. Thurber, Patterson, and Mount (2007) noted greater proportions of homesick among hospitalized than summer camp samples, with $50 \%$ greater or equal to the midpoint on the HS scale among the former, compared with $20 \%$ among the latter. Such factors as the relative (un)pleasantness of the experiences, reasons for leaving home, and selection factors may play a role in these contrasting proportions (e.g., parents and children have more choice to participate in summer camp than hospitalization).

How long does HS last? While one might assume HS to decline in intensity the longer the stay away, results have not been totally consistent with this expectation. Indeed, it may be oversimplistic to expect a linear decline, given that HS escalation has been found at certain holiday periods (van Vliet, 2001), and there may be different HS trajectories (cf. Vingerhoets, 2005). Some have reported no duration (reduction) effects at all (e.g., Watt \& Badger, 2009), while others reporting significant effects have evidenced relatively small ones and persistence of HS in some students (e.g., Stroebe et al., 2002). However, Brewin, Furnham, and Howes (1989) reported that $39 \%$ of first year students had experienced HS, which quickly declined, and others have similarly reported that HS diminishes during the first semester among students (Bell \& Bromnick, 1998). Ying (2005) reported a linear decline in HS among Taiwanese graduate students in the United States over the first 2 years of their stay. For most university students, it can probably be said that HS is a transient experience declining already in the first few weeks of being away. Van Vliet (2001) concluded: "the peak of the HS reaction is shortly after relocation and on average, the intensity of reported HS diminishes with time, after 16 months the level of HS is comparable to that previous to departure" (p. 122). Although the reasons for the discrepancies between studies remain somewhat unclear, they are probably at least partly because of differences in opportunities to visit or contact home and total duration of the stay away.

Those who remain homesick are probably the ones with high intensity HS experiences early on. Van Tilburg, Vingerhoets, van Heck, and Kirschbaum (1999) reported that of the "chronic" HS subgroup in their sample, 51\% still experienced HS after 3 years. Most adults with severe current HS retrospectively reported adverse HS experiences in childhood (van Tilburg, de Waal, Vingerhoets, \& van Heck, 1999). Likewise among younger persons: Thurber and colleagues (e.g., Thurber, 1999; Thurber, Sigman, Weisz, \& Schmidt, 1999) examined the course of HS in children and adolescents over summer camp periods. Those high in HS at the start remained high over their stay, others showed some improvement. For adults too, chronicity of HS has been related to its intensity (van Tilburg, Vingerhoets, \& van Heck, 1999). However, there may be different patterns for different subgroups. Among foreign employees, those who had been living abroad for 6-8 years had greater HS risk than newcomers (Eurelings-Bontekoe et al., 2000). The authors suggested several explanations for this apparent duration effect, but the cross-sectional nature of the investigation prevented firm conclusions from being drawn.

There may be cultural differences with respect to duration effects too, linked with special circumstances and sample characteristics. On the one hand, Tochkov, Levine, and Sanaka (2010) found that Indian students who remained for longer in the United States had greater HS (suggesting difficulties integrating in a strange culture). On the other hand, although a study of permanent immigration and therefore not for inclusion in the online supplemental material, a study by Tartakovsky (2007) of unaccompanied minor immigrants from Russia and the Ukraine to Israel found that HS in the third year was significantly less than in the first year, but among this select sample (they were apparently motivated, resourceful, and positive toward relocation), the level of HS was low in general.

Does HS commence before departure from home? There is some evidence that this may be the case. Findings of Thurber and colleagues (Thurber, 1999; Thurber et al., 1999) underline the need for further investigation, especially among children: They found childhood anticipation of HS before leaving home. Thurber (2005) included "anticipatory" methods of HS prevention before leaving home; some researchers have also included anticipation of leaving home in their HS descriptions (e.g., Fisher \& Hood, 1987; van Vliet, 2001), but most do not.

In conclusion: HS seems to be quite a common phenomenon, but it is difficult to be precise about prevalence or duration. The complex patterns of HS over time suggest the need for examination of different "trajectories" of HS (cf. Bonanno's trajectories of grief, e.g., Bonanno, 2004). One problem is that the patterns have frequently been confounded with relocation difficulties, which may follow a different course from separation difficulties. We also need better understanding of "peaks" in HS at different times; for example, who suffers from anticipatory HS; who from high but declining intensities; who from an increase over time away from home? In addition to the problems identified previously, the discrepancies regarding models and measures discussed earlier add to difficulties in assessing prevalence and duration of HS.

\section{Psychological and Physical Health Correlates and Consequences}

Endorsing the relationships with disturbances and disorders discussed earlier, the 55 studies suggest that homesick individuals can experience substantial distress and are at increased risk for psychological and physical health problems and lowered wellbeing. Notably, Thurber's work has shown that health consequences can be severe for children even at relatively short-term summer camps and that it can have clinically significant cognitive, emotional, and behavioral sequelae (e.g., Thurber, 2005). Healthrelated correlates of HS have been identified among older students too, again raising the question about the precise relationship of these symptoms with HS. For example, Fisher (1989) described HS as involving a sense of loneliness, dysphoria, emotional distress, depression, increased physical health problems, and ruminations about home. While physical health problems would seem a consequence, rumination about home seems integral to HS (i.e., a distinguishing feature of HS). In support of the latter, Bell and Bromnick (1998) regarded ruminative activity to be a distinguishing aspect of HS. Archer et al. (1998) included higher anxiety and somatic and obsessional symptoms to the range of symptoms. Similar correlates and consequences can be observed among other subgroups too, with some additional difficulties noted as well. For example, among transferred employees, early studies reviewed by Burt (1993) identified the possibility of increases in psychosomatic symptoms, as well as excesses in coronary heart disease risk. 
However, the possibility of third factors leading to higher risk of HS and such physical symptoms cannot be ruled out.

As the online supplemental material shows, there are indications that the impact of HS is broader than that on mental and physical health alone. HS has even been linked to Internet addiction (Ni, Yan, Chen, \& Liu, 2009). Perhaps less surprisingly, concentration among students is affected by HS (Burt, 1993). However, patterns are not always clear. To illustrate, there is some discrepancy as to whether HS affects academic performance. In contrast to earlier studies, van Vliet (2001) found that HS did not lead to poorer academic performance. High versus low HS groups had similar actual achievement and perceived academic achievement. Further investigation of this factor (including school and work performance), as well as related ones is called for. Adapting poorly to the new environment may be a consequence of HS (rather than integral to it), and various relocation adjustment difficulties have indeed been reported in association with HS (e.g., Fisher, 1989; van Vliet, 2001). Failure to cognitively assimilate new experiences when homesick is one example of this category of difficulties (cf., Bell \& Bromnick, 1998).

Of all the correlates and consequences of HS, one that is likely to have long-term repercussions, is that it can be so intense as to make being away from home unsustainable. In line with this, students with HS are 3 times more likely to drop out of college than those without (Thurber \& Walton, 2012). Unsustainability of HS could also lead to selection out of the most homesick from samples of people leaving home for shorter or longer stays: they may simply no longer go away at all.

Finally, an unusual, indirect consequence of HS has also been identified: HS may “. . . seriously hinder reintegration when back in the homeland, because of overidealization which makes the return often disappointing" (Vingerhoets, 2005, p. 3). This too merits further investigation.

Taken together, review of the available studies testifies to health consequences for some of those who experience HS. HS seems not just an "unfortunate, though familiar, vicissitude of life" (Davis, 1977 , p. 114), but rather, for some persons, to be a phenomenon encompassing varying degrees of suffering and ill health. Who, then, is most vulnerable to experiencing HS?

\section{Risk and Protective Factors}

Fisher (1989) demarked a wide range of vulnerabilities for HS. Thurber and Sigman (1998) reviewed empirical studies on risk and protective factors in childhood HS. They integrated predictors (14) and sequelae (8) of HS in children into a testable statistical model and found $69 \%$ of variance was accounted for by a "HS disposition" (negative interpersonal attitudes and perceived control) and little prior separation experience.

There has been continuing scientific effort to try to identify HS risk factors, and to understand why some people are affected by HS while others are not. We use the term "risk factor" to signify the situational, intrapersonal, and interpersonal characteristics associated with increased vulnerability to HS and its consequences (cf., Stroebe, Schut, \& Stroebe, 2007). Protective factors are those that appear to promote resilience and lower risk of adverse outcomes.

It is not easy to give a comprehensive overview of risk factors for HS (further information is available in the online supplemental material), because of the plethora of such factors and the possibility for complex interactions between variables. To illustrate, van Vliet (2001) in summing up risk factors based on his empirical work, covered multiple antecedent and concurrent variables, and a "recursive pattern . . . a self-amplifying loop" (p. 121). To highlight some of the main patterns of findings, we organize the factors into the following categories:

\section{Personality and Dispositional Factors}

One of the main lines of investigation of researchers in the HS field has been on personality factors, with a variety of them under scrutiny. Patterns of findings that emerge lead one to believe that a cluster of personality and dispositional factors play an important role in the occurrence of HS. Van Vliet (2001) linked emotional instability to HS, while the van Tilburg et al. studies (e.g., van Tilburg, Vingerhoets, \& van Heck, 1999, 1996d) have identified neuroticism and high anxiety as a critical feature: ". . . a personality-linked vulnerability factor is responsible for making anxious individuals prone to develop HS" (van Tilburg, Vingerhoets, van Heck, \& Kirschbaum, 1999, p. 189; see also Fisher, 1989). Introversion, rigidity, and low dominance levels have also been associated with HS (Eurelings-Bontekoe, Bontekoe, Vingerhoets, \& Fontijn, 1994), as have low self-esteem (Fisher, 1989), high levels of harm avoidance and low levels of self-directedness (Verschuur, Eurelings-Bontekoe, Spinhoven, \& Duijsens, 2003). In line with the findings for introversion and neuroticism, others have reported extraversion and emotional stability to be related to low HS (van Vliet, 2001).

There is little available information on the relationship between risk factors for assumed subdimensions of HS, but Beck et al.'s (2003) findings that sociotropy (cf. dependency) and autonomy were differentially related to the attachment versus dislike of university "facets of HS" (p. 162) are indicative. In support of our differentiation of attachment-to-home from current-environment dimensions, they suggest different ways that personality traits might affect coping with these two stressors, and differential vulnerabilities to each dimension. While the investigators consider both dimensions HS constructs, in our view, the differential patterns speak for separate theoretical treatment.

Not only general anxiety but relationship-specific anxiety has been investigated, with mixed results (more studies are needed). Some have looked into insecurity of attachment in relationship to HS. Kerns, Brumariu, and Abraham (2008) found no relationship between insecure attachment and HS among children at camp, in contrast to Thurber's studies as well as Stroebe et al. (2002) and Shal et al. (2011). But Thurber et al. (2007) did not find an association either among hospitalized children. Rather, HS was positively correlated with previous separation experiences (foster placement and weeks of previous separation from home) in the past. These children came from relatively chaotic home environments, with presumably unpleasant and uncontrollable separation experiences, that did not lead to positive expectations of separation. Little prior separation experience, however, was also associated with HS (Thurber \& Sigman, 1998), as well as strong family cohesion (Kazantzis \& Flett, 1998), and problematic parent-child relationships (van Tilburg, de Waal, et al., 1999). These factors were not defined as parent behavior, but may be because of parental overprotection or parental difficulty with the child's 
autonomy. In some studies, parent factors were unrelated to HS, such as parental separation anxiety in relation to hospitalized children (Thurber \& Walton, 2007).

Among older students, relationship-related factors have been associated with HS. Stroebe et al. (2002) found some evidence that attachment insecurity (in their Dutch sample) and conflict with parents (in their English one) were related to certain aspects of HS. Anxious attachment was also related to HS in a sample of Iranian male students (Shal et al., 2011). Van Vliet (2001, p. 84) did not find attachment dimensions to be strong predictors in his longitudinal study of students, but interestingly, in his expatriate study, he found a higher proportion (88\%) of securely attached persons in the expat group and only $1 \%$ were anxiously attached. The former percentage is higher, the latter lower than is typically found in the literature (p. 109). This again suggests selection: that persons who are insecure, particularly the anxiously attached, may not enter expatriate work to begin with. Other relationship factors may be important too: Brewin et al. (1989) and Carden and Feicht (1991) found dependency on others to be a HS risk factor. One study investigated parenting styles, and found that students raised by authoritarian or uninvolved parents were less likely to experience HS than students raised by authoritative or permissive parents (Nijhof \& Engels, 2007). So there is some evidence that the nature of the bonds that individuals hold toward close others and their (early) relationships may underlie HS. Clearly, further exploration among different subgroups would be useful here too, such as youth and adult samples.

Various more general, socially oriented factors may be significant too. For children, Kerns et al. (2008) suggested that a positive social self-concept and relationships with peers may be HS protective factors. With university students, Watt and Badger (2009) provided preliminary (experimental) evidence that belongingness needs (an innate drive for a minimum number of lasting interpersonal relationships) are threatened on relocation, and are a cause of HS. Interestingly, and in contrast to earlier claims, Watt and Badger (2009) found that friendship and social support substitution in new environment did not compensate for those left behind or reduce HS. Examining a slightly different support aspect, Eurelings-Bontekoe et al. (1994) found that high levels of social support seeking among the military and expression of emotions and seeking social support among adults were found to be characteristic of severe HS (Verschuur et al., 2001). It is interesting, though, among students, over the course of their first semester, high self-disclosers experienced a significantly greater reduction in HS than low disclosers (Bell \& Bromnick, 1998). Furthermore, Newland and Furnham (1999) found that low levels of perceived social support, combined with a high level of psychological disturbance were associated with HS. Van Vliet (2001) also reported that seeking social support has beneficial effects on HS. These apparent discrepancies may have to do with the particular subgroup and separation experience in question, as well as the precise content of what is shared with others, or with the particular type of support which is sought (e.g., one would surmise that high scores on sharing ruminative thoughts about home would be associated with HS, while seeking social support in general might have the opposite effect, perhaps through processes of integration or distraction).

Other coping mechanisms can be considered among personality and dispositional factors. Thurber and colleagues have examined coping processes among children in relationship to HS in particular detail. Thurber and Weisz (1997b), for example, found that effective coping occurred through distractive activities, and through physical activity among children and adolescents. Van Vliet (2001, p. 83) found that problem focused coping was effective for students (as well as seeking social support, as reported earlier), while self-blame had detrimental effects. A coping style of focusing on the positive had an intensifying effect, leading to a higher level of HS and longer duration of HS. Grief research may give further leads as to how research on (in)effective coping strategies can be usefully expanded (e.g., ruminative processes; emotion regulation \& control, cf. Stroebe et al., 2007).

Finally, two further dispositional features seem worth highlighting. First, investigators have pointed to the importance of the controllability variable (Burt, 1993; Fisher, 1989; Fisher, Murray, \& Frazer, 1985; Flett et al., 2009; Thurber, 1999; Thurber \& Weisz, 1997a, 1997b). Studies have suggested that when one's own perceived controllability over the decision to relocate is low or minimal, HS risk is higher (Fisher, 1989; Flett et al., 2009). Second, an unusual feature for the HS area-self-compassionwas noted by Terry, Leary, and Mehta (2013) as a moderator of HS in transition to college. Those high in self-compassion had lower HS.

\section{Sociodemographic Factors}

Do some life circumstances and subgroup characteristics increase the risk of HS? In this category too, diverse variables have been investigated. One of the most salient for many people is the question of gender differences. Lay beliefs may lead to the expectation that females experience greater HS. But review of the literature does not unequivocally confirm this, with some studies reporting similar prevalences among males and females, including children (e.g., Brewin et al., 1989; Fisher \& Hood, 1987; Scopelliti \& Tibero, 2010; Thurber et al., 2007: hospitalized children), while others (e.g., Archer et al., 1998; Stroebe et al., 2002) find higher HS among female students. Gender differences in ways of coping with HS were reported by Brewin et al. (1989), with women seeking more social support than men. Similarly, Archer et al. (1998) found higher levels of intrusive thinking about HS among women. A rare study of a different ethnic group actually reported more HS among Indian men than women students in the United States (Tochkov et al., 2010).

Age groups vary considerably across the studies reviewed in the online supplemental material, but there is not a lot of information available on any age differences in HS. Some have reported no age differences in HS (e.g., Fisher \& Hood, 1987). Others have pointed out that finding differences depends on the age range included in the studies. Among children, for example, Thurber (1995) found younger ones to be more vulnerable to HS; Kerns et al. (2008) did not, but in a narrower age range. Thurber and Weisz (1997a) made the case that older boys may deal better with HS because of age-related changes in coping. To our knowledge, there has not been any comparison in HS across the broad life span, just some limited age-range comparisons. For example, like the pattern for children, younger college student nurses have been found to suffer from HS more than older ones (Porritt \& Taylor, 1981). Looking at the relationship of HS with age differently: Those who experience HS at a younger age are more prone to it later in life (e.g., 
Eurelings-Bontekoe et al., 1994; Verschuur et al., 2001). So what about differences between childhood and adulthood in HS in general? It is difficult to make comparisons as these groups differ on so many age-related dimensions (e.g., the freedom of choice to stay home; natural dependence on adult care givers; availability of coping strategies; admission and expression of emotions), and there is a lack of longitudinal studies from childhood to adulthood. But it is noteworthy that Thurber and Walton (2012) regard most risk and protective factors among students as being identical to those for childhood HS: in this respect, HS susceptibility may not be discriminant across age groups.

Turning to other sociodemographic variables: It would seem reasonable to assume that greater distance from home would increase the likelihood of HS, but here again, there are some discrepancies in the literature. Brewin et al. (1989) found no significant difference according to distance from home but Fisher et al. (1985) found that those who were further away experienced greater HS, as did Stroebe et al. (2002) on the missing of family subscale.

Are there cultural differences in HS? Although there are reasons to believe that HS is in some respects a universal phenomenon, as the online supplemental material shows, few investigations have been conducted outside industrialized societies. Carden and Feicht (1991) compared HS among Turkish and American students in their own countries; Hojat and Herman (1985) studied a sample of Iranian and Filipino individuals (all resident physicians in the United States). Lu (1990) studied Chinese students at United Kingdom universities. HS was a commonly reported phenomenon across these various studies. Some differences in HS were reported for the cultural groups within studies. Poyrazli and Lopez (2007) found that international students in the United States were more homesick than American students, with younger age, lack of English proficiency, and higher perceived discrimination levels predicting HS. Stroebe et al. (2002) using the same HS measure across 2 cultures, found different patterns of HS within Europe. For instance, students from the United Kingdom were more homesick than Dutch ones and females from the United Kingdom suffered more than any other group. Taken together, the results suggest that HS is indeed evident across cultures, but there may be some culturally specific patterns/differences. Like many other areas of cross-cultural research, such as the somewhat comparable bereavement-separation area (cf. Rosenblatt, 2013), it is difficult to compare the prevalence and experience of HS when trying to understand emotions and cognitions in cultures very different from our own, and when studies differ in their use of HS measures, design features, and so on.

\section{Conclusions}

How should research on risk factors proceed? The range of variables could usefully be extended. For example, examination of the role of religiousness in protecting against HS seems hardly to have been investigated (cf., Longo \& Kim-Spoon, 2013). However, rather than looking at a single or few factors, larger scale, longitudinal studies need to examine differential predictability of variables. Although rare so far, there are some examples of such strategies in the literature, including the integration of risk/protective factors by Thurber and Sigman (1998, as mentioned under the 'Risk and Protective Factors' section). Van Vliet (2001, Chapter 6) also examined multiple predictors and the extent to which different factors accounted for variance, and Flett et al. (2009) suggested the need for a "multidimensional interactionism model"; that is, one that would explore the role of dispositional and situational-specific coping factors and resources. Second, we need to further distinguish causal versus exacerbating factors and to link these to specific consequences. Perhaps most important, at least in the current context, differentiation is needed regarding which factors are predictors of missing home and loved ones versus maladjustment to the new environment.

\section{Prevention and Treatment of HS}

Averill and Sundararajan (2014, p. 19) recount words seen on a badge in a souvenir shop in Taiwan: "Transform the grief of separation to nostalgia." Is it possible to turn the pain of HS into something less harrowing, through professional intervention? ${ }^{1} \mathrm{Un}$ til recent decades it was quite commonly believed that there was no "cure" but to return home, somewhat echoed by van Vliet (2001): "The most simplistic prevention strategy for HS is not to leave home, the most simplistic treatment for HS is to return home" (p. 122). But considerable effort has been invested in developing intervention programs for homesick individuals during their sojourns away from home (and sometimes before leaving), and the general consensus has shifted toward viewing HS as no longer unsustainable. There is some empirical basis for this. Even though quite a number of articles describe strategies in prevention and treatment, we found just four studies actually empirically evaluating interventions (Nejad, Pak, \& Zarghar, 2013; Pennebaker, Colder, \& Sharp, 1990; Tavakoli, Lumley, Hijazi, SlavinSpenny, \& Parris, 2009; Thurber, 2005).

Much of the recent research on intervention has been conducted by Thurber and his colleagues, with concentration on children and adolescents, and also college students (for summaries, see Thurber $\&$ Walton, 2007, 2012). Their approach covers prevention strategies as well as intervention. For example, Thurber (2005) described a multimodal HS prevention study for boys at 2-week summer camp. Furthermore, Thurber and Walton (2012) reviewed new research that points to promising prevention and treatment strategies for HS, stressing the need for effectiveness research. They have identified processes that could be addressed in prevention and treatment. After examining the course/occurrence of HS across time, Thurber and Weisz (1997b) concluded that effective coping among children and adolescents is furthered by distractive activities and physical activity, which is in line with Fisher's (1989) statement that HS is experienced more during passive mental tasks than active physical ones.

A variety of other strategies for preventing and treating HS can be found in the literature. Fisher (1990) promoted her attentional management strategy, which promotes the "restructuring of the qualities, opportunities and facilities offered by the new environment (p. 307)". As such, the emphasis is on the relocation rather than HS. Van Tilburg et al. (1997) suggested the need to focus on

\footnotetext{
${ }^{1}$ It is interesting that recent work suggests that nostalgia-a largely positive emotional experience-may have the capacity to prevent HS related negative feelings (cf. Routledge, Wildschut, Sedikides, \& Juhl, 2013). This underlines the idea that interventions may need to focus on home-related issues too (we return to this later).
} 
(in)effective coping strategies which can be used to guide the development of adequate interventions for HS individuals. Nejad et al. (2013) investigated the effectiveness of 9 sessions of social skills training for HS in female students, finding this treatment more effective than a control group. Tavakoli et al. (2009) offered different interventions in a randomized trial (assertiveness training, expressive writing, both or wait-list control group) for international students (apparently not selected for maladjustment, but open to all). Notably, expressive writing led to higher HS. Possibly, the content was ruminative about home (they shared emotionally difficult experiences related to home and current locations). Pennebaker et al. (1990) also found that HS decreased in controls by the end of the first semester, whereas this was not the case when students wrote about their thoughts and feelings. However, by the end of the year, students who participated in the writing exercises were less homesick.

Researchers have made efforts to provide guidance for professionals coming into contact with HS. Thurber et al. (2007) give tips for HS prevention for hospital staff, and for parents/caregivers (see pp. 22-23). Baier and Welch (1992) describe nursing interventions (which are not empirically based). There is much that can be gleaned for health care professionals in general, although there are limits to what certain strategies can achieve. For example, Watt and Badger's (2009) examined the impact of contacting home while homesick (p. 527): They found that phoning home does not reduce HS, but serves to "act as a comfort when HS is strong" (p. 527).

The conclusions of van Tilburg (2012, personal communication), deserve further investigation: "Distraction is much more preferred over contact with or thinking about home. A place for thoughts about home should be reserved for times when we feel good. In times when we feel bad, we need the safety of home the most and thus relinquish to our feelings, but it serves us best to become oriented on the new environment: "What is fun here?" "How can I beat loneliness, sadness, boredom in my new environment?" This increases self-efficacy, social support (in the new environment), and adjustment"

It is beyond the scope of the current article to expand on prevention and treatment techniques, but we do need to consider them in the context of their home-new place focus: To what extent do HS prevention and treatment guidelines follow the dualmissing familiar and maladjustment to new environment versus focusing only on the one or the other? Differences in approach are evident already from the previous descriptions. Thurber's approach typically incorporates focus on both aspects. For example, his "Multimodal Homesickness Prevention Package" (Thurber, 2005) includes both. It combines information/familiarization with the new environment with psycho-education about HS (for family and staff as well), guidance toward using coping strategies that reduce HS. By contrast, Fisher $(1989,1990)$ has emphasized adjustment to the new environment and the development of skills and management strategies for coping with this relocation (considering HS as rather symptomatic of maladjustment to the new environment). Indeed, two intervention programs focusing on assertiveness/social skills in students have been found to positively influence HS (Nejad et al., 2013; Tavakoli et al., 2009).

It is important to note that HS is regarded as fundamentally a missing home phenomenon, with associated and interacting adjustment difficulties. Therefore, prevention and intervention strat- egies should contain elements directed at the maladjustment to the new environment, as well as elements focused on dealing with missing home: There are good reasons to include treatment relating to maladjustment to the new environment, in prevention or treatment programs, given that these difficulties can interact/be correlated with HS and/or consequences of it. Nevertheless, issues about missing home should not be ignored but integral (e.g., thinking about positive/negative home features for a limited time as well as distraction from these and focus on new place issues). Thurber's (2005) approach is exemplary of a dual one, in that attention to the two dimensions is clearly identifiable and integrated in his training program.

\section{Future Directions/Closing Remarks}

As advocated in earlier sections of this article, in our view a major step forward in the scientific study of HS would be for researchers to focus on HS as a separation experience and "tease out" specific patterns, correlates and consequences associated with this phenomenon, separately from difficulties associated with adjustment to a new environment. Such an approach would follow the narrow definition of HS as a "minigrief." It would restrict measurement instruments of HS per se to separation items, while separately and specifically also mapping associated adjustment difficulties and other correlates. It would enable results of studies to be more easily compared.

Although we systematically reviewed the body of empirical studies, the conclusions that we were able to draw have frequently been tentative. In addition to the need for a narrower, theoryguided approach, limitations of studies have become evident. These include the predominance of cross-sectional investigations; longitudinal studies are quite rare. Researchers (e.g., van Tilburg, personal communication) have noted problems obtaining funding for HS research. The fact that HS is not a diagnostic category of mental disorders probably limits interest in the topic, despite the range and severity of HS's consequences. How can such difficulties in conducting HS research be overcome? Future research may follow two lines of approach. First, reasonably short HS questionnaires can be added to large-scale, longitudinal cohort studies. Second, it may be worth revisiting older, longitudinal data sets in the light of the patterns of findings emerging from this review, and ones deriving from our specific perspective (e.g., separating the "home" from "new place" focus and examining differential predictors: cf. Stroebe et al., in press). In addition to these strategies, use of other (nonquestionnaire) approaches and extension to novel domains could also add to understanding. Experimental studies such as that of Watt and Badger (2009) can tease out mechanisms, mediators, and moderators of the different HS domains. Research on biological markers and physiological measurement of HS have also only recently begun. One potential direction could be to examine psychobiological changes accompanying leaving and returning to home. This could further test the usefulness of separate consideration of the home-away dimensions. Furthermore, we still know little about the origins of HS in terms of genetic versus environmental causes. In this context, it is important to note that Scaini, Ogliari, Eley, Zavos, and Battaglia (2012) and RobersonNay, Eaves, Hettema, Kendler, and Silberg (2012) have proposed a genetic component in separation anxiety. Another channel for future investigation would be systematic demarcation of HS from 
other conditions. The field now needs to identify comorbidity, precipitating and exacerbating conditions in relationship to missing-home separately from new-place-adaptation. As a final suggestion, exploration of the role of modern technology seems called for. For example, do contemporary communication techniques help prevent HS, by facilitating contact with home? Or: Do the additional visual aspects of such facilities as Skype or Facetime make a difference in reducing HS? Returning to the difficulty in conducting HS research: Use of Internet hopefully opens up opportunities for further scientific investigation of HS, just as it has in the bereavement-grief area (cf. Eisma, 2015).

There is room for other types of expansion. Samples in HS research have largely been limited to children/adolescents and young adults (with many student samples). There are good reasons for this, because these are vulnerable subgroups, with many leaving home for the first time during these periods in their lives. Nevertheless, we have indicated additional vulnerable samples which need further investigation (e.g., soldiers, expatriates) and some subgroups have been largely neglected. For example, Agterberg and Passchier (1998) identified HS as a major problem for seamen, as reported to harbor physicians in a small qualitative study. The title of an earlier article "Mentally handicapped children get homesick too" (Dopson, 1980) suggests that these children, when away from home, may be an underacknowledged subgroup vulnerable to HS. Likewise, children placed in residential or foster care (e.g., to safeguard them from abuse or neglect) may be prone to HS. Shechory and Sommerfeld (2007) looked at behavioral problems of children in residential care but HS may feature prominently too, particularly among recent (including adult) residential care dwellers. Furthermore, most but not all studies have been conducted within western, industrial societies, and again, extension to other countries is called for. It is interesting to note the impact of cultural factors on selection of children's samples even within western studies, with boarding schoolchildren predominating in samples from the United Kingdom, while those at summer camps are the focus in U.S. investigations. Another useful line for future research would be to extend investigation to those who have experienced HS in the past and avoid any further experience of separation from home. Indeed, Thurber (1999) found selection out of repeating separation experiences among high HS boys. Finally, it would be informative to focus on groups that have never experienced HS and to establish how they differ from HS groups, or further examine HS history of those not currently in a separation situation, to inventorize past experiences and reactions, and assess risk of future HS.

Outstanding questions also remain (cf. Vingerhoets, 2005, p. 4). For example: How early in life can one become homesick? How does one become so strongly attached to one's home, as well as those who live there, that one suffers extreme HS on leaving? What is the role of parental behavior in separation situations? Can HS be considered chronic and if so, under what circumstances? Can it be "cured"? Is professional intervention adequate? Partial answers to some questions have been given in this review of the HS literature. We consider it important to establish conceptual clarity with respect to HS. While empirical evidence has shown that most people adapt well to leaving home, for a few, the impact can be devastating. Moreover, we restricted our review to the relatively benign experience of temporary stays away from home: Hopefully, knowledge can be applied to help understand the more complex adjustment processes of those who cannot return home, as in cases of permanent emigration from a war-torn homeland.

\section{References}

Agterberg, G., \& Passchier, J. (1998). Stress among seamen. Psychological Reports, 83, 708-710. http://dx.doi.org/10.2466/pr0.1998.83.2.708

American Psychiatric Association. (2013). Diagnostic and statistical manual of mental disorders (5th ed.). Washington, DC: Author.

Archer, J., Ireland, J., Amos, S. L., Broad, H., \& Currid, L. (1998). Derivation of a homesickness scale. British Journal of Psychology, 89, 205-221. http://dx.doi.org/10.1111/j.2044-8295.1998.tb02681.x

Averill, J., \& Sundararajan, L. (2014). Experiences of solitude: Issues of assessment, theory, and culture. In R. Coplan \& J. Bowker (Eds.), A handbook of solitude: Psychological perspectives on social isolation, social withdrawal, and being alone (pp. 90-108). Hoboken, NJ: WileyBlackwell.

Baier, M., \& Welch, M. (1992). An analysis of the concept of homesickness. Archives of Psychiatric Nursing, 6, 54-60. http://dx.doi.org/ 10.1016/0883-9417(92)90055-N

Baker, R. W., \& Siryk, B. (1999). Student Adaptation to College Questionnaire. Los Angeles, CA: Western Psychological Services.

Beck, R., Taylor, C., \& Robbins, M. (2003). Missing home: Sociotropy and autonomy and their relationship to psychological distress and homesickness in college freshmen. Anxiety, Stress, \& Coping: An International Journal, 16, 155-166. http://dx.doi.org/10.1080/10615806.2003 10382970

Bell, J., \& Bromnick, R. (1998). Young people in transition: The relationship between homesickness and self-disclosure. Journal of Adolescence, 21, 745-748. http://dx.doi.org/10.1006/jado.1998.0193

Bonanno, G. A. (2004). Loss, trauma, and human resilience: Have we underestimated the human capacity to thrive after extremely aversive events? American Psychologist, 59, 20-28. http://dx.doi.org/10.1037/ 0003-066X.59.1.20

Brewin, C., Furnham, A., \& Howes, M. (1989). Demographic and psychological determinants of homesickness and confiding among students. British Journal of Psychology, 80, 467-477. http://dx.doi.org/10.1111/ j.2044-8295.1989.tb02336.x

Burt, C. (1993). Concentration and academic ability following transition to university: An investigation of the effects of homesickness. Journal of Environmental Psychology, 13, 333-342. http://dx.doi.org/10.1016/ S0272-4944(05)80255-5

Carden, A., \& Feicht, R. (1991). Homesickness among American and Turkish college students. Journal of Cross-Cultural Psychology, 22, 418-428. http://dx.doi.org/10.1177/0022022191223007

Davis, F. (1977). Nostalgia, identity, and the current nostalgia wave. Journal of Popular Culture, 11, 414-424. http://dx.doi.org/10.1111/j .0022-3840.1977.00414.x

de Vries, M. (Ed.). (1992). The experience of psychopathology: Investigating mental disorders in their natural settings. New York, NY: Cambridge University Press. http://dx.doi.org/10.1017/CBO9780511663246

Dopson, L. (1980). Mentally handicapped children get homesick too. Nursing Times, 76, 1867.

Eisma, M. (2015). Rumination following bereavement: Assessment, working mechanisms and intervention. $\mathrm{PhD}$ thesis, Utrecht University. Utrecht, the Netherlands: Ridderprint BV.

Eurelings-Bontekoe, E., Brouwers, E., \& Verschuur, M. (2000). Homesickness among foreign employees of a multinational high-tech company in The Netherlands. Environment and Behavior, 32, 443-456. http://dx.doi.org/10.1177/00139160021972612

Eurelings-Bontekoe, E., Brouwers, E., Verschuur, M., \& Duijsens, I. (1998). DSM-III-R and CD-10 personality disorder features among women experiencing two types of self-reported homesickness: An exploratory study. British Journal of Psychology, 89, 405-416. http://dx .doi.org/10.1111/j.2044-8295.1998.tb02693.x 
Eurelings-Bontekoe, E., Duijsens, I., \& Verschuur, M. (1996a). Prevalence of DSM-III and ICD-10 personality disorders among military conscripts suffering from homesickness. Personality and Individual Differences, 21, 431-440. http://dx.doi.org/10.1016/0191-8869(96)00031-1

Eurelings-Bontekoe, E., Tolsma, A., Verschuur, M., \& Vingerhoets, A. (1996b). Construction of a homesickness questionnaire using a female population with two types of self-reported homesickness. Preliminary results. Personality and Individual Differences, 20, 415-421. http://dx .doi.org/10.1016/0191-8869(95)00210-3

Eurelings-Bontekoe, E., Verschuur, M., Koudstaal, A., van der Sar, S., \& Duijsens, I. (1995). Construction of a homesickness-questionnaire. Preliminary results. Personality and Individual Differences, 19, 319-325. http://dx.doi.org/10.1016/0191-8869(95)00056-C

Eurelings-Bontekoe, E., Vingerhoets, A., \& Fontijn, T. (1994). Personality and behavioral antecedents of homesickness. Personality and Individual Differences, 16, 229-235. http://dx.doi.org/10.1016/01918869(94)90161-9

Fisher, S. (1989). Homesickness, cognition and health. Hove, United Kingdom: Laurence Erlbaum.

Fisher, S. (1990). Helping the homesick: Attentional management strategy, commitment and adaptation. In S. Fisher \& C. Cooper (Eds.), On the move: The psychology of change and transition (pp. 305-314). Chichester, United Kingdom: Wiley.

Fisher, S., Elder, L., \& Peacock, G. (1990). Homesickness in a school in the Australian bush. Children's Environments Quarterly, 7, 15-22. Retrieved from http://www.jstor.org/stable/41514740

Fisher, S., \& Hood, B. (1987). The stress of the transition to university: A longitudinal study of psychological disturbance, absent-mindedness and vulnerability to homesickness. British Journal of Psychology, 78, 425441. http://dx.doi.org/10.1111/j.2044-8295.1987.tb02260.x

Fisher, S., Murray, K., \& Frazer, N. (1985). Homesickness, health, and efficiency in first year students. Journal of Environmental Psychology, 5, 181-195. http://dx.doi.org/10.1016/S0272-4944(85)80016-5

Flett, G., Endler, N., \& Besser, A. (2009). Separation anxiety, perceived controllability, and homesickness. Journal of Applied Social Psychology, 39, 265-282. http://dx.doi.org/10.1111/j.1559-1816.2008.00438.x

Green, P. (2011). The enemy within? Explorations of personhood, friendship and difference amongst Brazilians living in Japan. Journal of Ethnic and Migration Studies, 37, 373-388. http://dx.doi.org/10.1080/ 1369183X.2011.526779

Hansson, A., Clausson, E., \& Janlöv, A. C. (2012). International school children's health needs: School nurses' views in Europe. The Journal of School Nursing, 28, 144-152. http://dx.doi.org/10.1177/ 1059840511425679

Hojat, M., \& Herman, M. W. (1985). Adjustment and psychosocial problems of Iranian and Filipino physicians in the U.S. Journal of Clinical Psychology, 41, 130-136. http://dx.doi.org/10.1002/ 1097-4679(198501)41:1<130::AID-JCLP2270410123>3.0.CO;2-K

Ireland, C., \& Archer, J. (2000). Homesickness among a prison population. Legal and Criminological Psychology, 5, 97-106. http://dx .doi.org/10.1348/135532500168001

Kazantzis, N., \& Flett, R. (1998). Family cohesion and age as determinants of homesickness in university students. Social Behavior and Personality, 26, 195-202. http://dx.doi.org/10.2224/sbp.1998.26.2.195

Kerns, K., Brumariu, L., \& Abraham, M. (2008). Homesickness at summer camp: Associations with the mother-child relationship, social selfconcept, and peer relationships in middle childhood. Merrill-Palmer Quarterly, 54, 473-498. http://dx.doi.org/10.1353/mpq.0.0010

Longo, G., \& Kim-Spoon, J. (2013). Homesickness in college students: The role of religion in combating depression. Mental Health, Religion \& Culture, 16, 489-500. http://dx.doi.org/10.1080/13674676.2012.696600

Lu, L. (1990). Adaptation to British universities: Homesickness and mental health of Chinese students. Counselling Psychology Quarterly, 3, 225232. http://dx.doi.org/10.1080/09515079008254253
Nawijn, J. (2010). The holiday happiness cure: A preliminary investigation into mood during a holiday abroad. International Journal of Tourism Research, 12, 281-290. http://dx.doi.org/10.1002/jtr.756

Nejad, S., Pak, S., \& Zarghar, Y. (2013). Effectiveness of social skills training in homesickness, social intelligence and interpersonal sensitivity in female university students resident in dormitory. International Journal of Psychology and Behavioral Research, 2, 168-175.

Newland, J., \& Furnham, A. (1999). Perceived availability of social support. Personality and Individual Differences, 27, 659-663. http://dx.doi .org/10.1016/S0191-8869(98)00254-2

Ni, X., Yan, H., Chen, S., \& Liu, Z. (2009). Factors influencing internet addiction in a sample of freshmen university students in China. Cyberpsychology \& Behavior, 12, 327-330. http://dx.doi.org/10.1089/cpb .2008 .0321

Nijhof, K. S., \& Engels, R. C. (2007). Parenting styles, coping strategies, and the expression of homesickness. Journal of Adolescence, 30, 709 720. http://dx.doi.org/10.1016/j.adolescence.2006.11.009

Pedersen, E. R., Neighbors, C., Larimer, M. E., \& Lee, C. M. (2011). Measuring Sojourner Adjustment among American students studying abroad. International Journal of Intercultural Relations, 35, 881-889. http://dx.doi.org/10.1016/j.ijintrel.2011.06.003

Pennebaker, J. W., Colder, M., \& Sharp, L. K. (1990). Accelerating the coping process. Journal of Personality and Social Psychology, 58, 528-537. http://dx.doi.org/10.1037/0022-3514.58.3.528

Porritt, D., \& Taylor, D. (1981). An exploration of homesickness among student nurses. Australian and New Zealand Journal of Psychiatry, 15 57-62. http://dx.doi.org/10.3109/00048678109159411

Poyrazli, S., \& Lopez, M. D. (2007). An exploratory study of perceived discrimination and homesickness: A comparison of international students and American students. The Journal of Psychology: Interdisciplinary and Applied, 141, 263-280. http://dx.doi.org/10.3200/JRLP.141.3 $.263-280$

Radcliffe, J., \& Turk, V. (2008). Distress in children with learning disabilities at a respite unit: Perspectives on their experiences. British Journal of Learning Disabilities, 36, 91-101. http://dx.doi.org/10.1111/j.14683156.2007.00469.x

Roberson-Nay, R., Eaves, L. J., Hettema, J. M., Kendler, K. S., \& Silberg, J. L. (2012). Childhood separation anxiety disorder and adult onset panic attacks share a common genetic diathesis. Depression and Anxiety, 29, 320-327. http://dx.doi.org/10.1002/da.21931

Rosenblatt, P. (2013). Family grief in cross-cultural perspective. Family Science, 4, 12-19. http://dx.doi.org/10.1080/19424620.2013.819226

Routledge, C., Wildschut, T., Sedikides, C., \& Juhl, J. (2013). Nostalgia as a resource for psychological health and well-being. Social and Personality Psychology Compass, 7, 808-818. http://dx.doi.org/10.1111/spc3 12070

Sandhu, D. S., \& Asrabadi, B. R. (1994). Development of an acculturative stress scale for international students: Preliminary findings. Psycholog ical Reports, 75, 435-448. http://dx.doi.org/10.2466/pr0.1994.75.1.435

Scaini, S., Ogliari, A., Eley, T. C., Zavos, H. M., \& Battaglia, M. (2012) Genetic and environmental contributions to separation anxiety: A metaanalytic approach to twin data. Depression and Anxiety, 29, 754-761. http://dx.doi.org/10.1002/da.21941

Scopelliti, M., \& Tibero, L. (2010). Homesickness in university students: The role of multiple place attachment. Environment and Behavior, 42, 335-350. http://dx.doi.org/10.1177/0013916510361872

Shal, R., Sharbaf, H. A., Abdeekhodaee, M. S., Masoleh, S. M., \& Salehi, I. (2011). Survey the relationship between attachment style and general self efficacy with homesickness among college students. Procedia: Social and Behavioral Sciences, 30, 538-541. http://dx.doi.org/10.1016/ j.sbspro.2011.10.105

Shechory, M., \& Sommerfeld, E. (2007). Attachment style, home-leaving age and behavioral problems among residential care children. Child 
Psychiatry and Human Development, 37, 361-373. http://dx.doi.org/ 10.1007/s10578-007-0051-z

Shin, H., \& Abell, N. (1999). The Homesickness and Contentment Sale: Developing a culturally sensitive measure of adjustment for Asians. Research on Social Work Practice, 9, 45-60. http://dx.doi.org/10.1177/ 104973159900900104

Smrcek, M., \& Stiksrud, H. (1994). Commitment and homesickness during post-adolescence. Studia Psychologica, 36, 211-221.

Steiner, A. R., \& Coan, J. A. (2011). Prefrontal asymmetry predicts affect, but not beliefs about affect. Biological Psychology, 88, 65-71. http://dx .doi.org/10.1016/j.biopsycho.2011.06.010

Stroebe, M., Schut, H., \& Nauta, M. (in press). Is homesickness a minigrief? Clinical Psychological Science.

Stroebe, M., Schut, H., \& Stroebe, W. (2007). Health outcomes of bereavement. The Lancet, 370, 1960-1973. http://dx.doi.org/10.1016/S01406736(07)61816-9

Stroebe, M., van Vliet, T., Hewstone, M., \& Willis, H. (2002). Homesickness among students in two cultures: Antecedents and consequences. British Journal of Psychology, 93, 147-168. http://dx.doi.org/10.1348/ 000712602162508

Tartakovsky, E. (2007). A longitudinal study of acculturative stress and homesickness: High-school adolescents immigrating from Russia and Ukraine to Israel without parents. Social Psychiatry and Psychiatric Epidemiology, 42, 485-494. http://dx.doi.org/10.1007/s00127-0070184-1

Tavakoli, S., Lumley, M. A., Hijazi, A. M., Slavin-Spenny, O. M., \& Parris, G. P. (2009). Effects of assertiveness training and expressive writing on acculturative stress in international students: A randomized trial. Journal of Counseling Psychology, 56, 590-596. http://dx.doi.org/ $10.1037 / \mathrm{a} 0016634$

Terry, M., Leary, M., \& Mehta, S. (2013). Self-compassion as a buffer against homesickness, depression, and dissatisfaction in the transition to college. Self and Identity, 11, 1-13.

Thurber, C. (1995). The experience and expression of homesickness in preadolescent and adolescent boys. Child Development, 66, 1162-1178. http://dx.doi.org/10.2307/1131805

Thurber, C. A. (1999). The phenomenology of homesickness in boys. Journal of Abnormal Child Psychology, 27, 125-139. http://dx.doi.org/ 10.1023/A:1021911514768

Thurber, C. A. (2005). Multimodal homesickness prevention in boys spending 2 weeks at a residential summer camp. Journal of Consulting and Clinical Psychology, 73, 555-560. http://dx.doi.org/10.1037/0022006X.73.3.555

Thurber, C., Patterson, D., \& Mount, K. (2007). Homesickness and children's adjustment to hospitalization: Toward a preliminary model. Children's Health Care, 36, 1-28. http://dx.doi.org/10.1080/ 02739610701316753

Thurber, C. A., \& Sigman, M. D. (1998). Preliminary models of risk and protective factors for childhood homesickness: Review and empirical synthesis. Child Development, 69, 903-934. http://dx.doi.org/10.1111/j .1467-8624.1998.tb06151.x

Thurber, C. A., Sigman, M. D., Weisz, J. R., \& Schmidt, C. K. (1999). Homesickness in preadolescent and adolescent girls: Risk factors, behavioral correlates, and sequelae. Journal of Clinical Child Psychology, 28, 185-196. http://dx.doi.org/10.1207/s15374424jccp2802_6

Thurber, C. A., \& Walton, E. A. (2007). Preventing and treating homesickness. Child and Adolescent Psychiatric Clinics of North America, 16, 843-858. http://dx.doi.org/10.1016/j.chc.2007.05.003

Thurber, C. A., \& Walton, E. A. (2012). Homesickness and adjustment in university students. Journal of American College Health, 60, 415-419. http://dx.doi.org/10.1080/07448481.2012.673520

Thurber, C., \& Weisz, J. (1997a). Describing boys' coping with homesickness using a two-process model of control. Anxiety, Stress and Coping:
An International Journal, 10, 181-202. http://dx.doi.org/10.1080/ 10615809708249300

Thurber, C. A., \& Weisz, J. R. (1997b). "You can try or you can just give up": The impact of perceived control and coping style on childhood homesickness. Developmental Psychology, 33, 508-517. http://dx.doi .org/10.1037/0012-1649.33.3.508

Tochkov, K., Levine, L., \& Sanaka, A. (2010). Variation in the prediction of cross-cultural adjustment by Asian-Indian students in the United States. College Student Journal, 44, 677-689.

van Tilburg, M., \& Vingerhoets, A. (Eds.). (2005). Psychological aspects of geographic moves: Homesickness and acculturation. Tilburg, the Netherlands: Tilburg University Press.

van Tilburg, M. A., Vingerhoets, A. J., \& van Heck, G. L. (1996) Homesickness: A review of the literature. Psychological Medicine, 26, 899-912. http://dx.doi.org/10.1017/S0033291700035248

van Tilburg, M., Vingerhoets, A., \& van Heck, G. (1997). Coping with homesickness: Construction of the Adult Homesickness Coping Questionnaire (AHCQ). Personality and Individual Differences, 22, 901-907. http://dx.doi.org/10.1016/S0191-8869(97)00010-X

van Tilburg, M., de Waal, K., Vingerhoets, A., \& van Heck. (1999). Homesickness and separation anxiety: Are they different? Psychological Studies, 44, 95-100.

van Tilburg, M., Vingerhoets, A., \& van Heck, G. (1999). Determinants of homesickness chronicity: Coping and personality. Personality and Individual Differences, 27, 531-539. http://dx.doi.org/10.1016/S01918869(98)00262-1

van Tilburg, M., Vingerhoets, A., van Heck, G., \& Kirschbaum, C. (1999). Homesickness, mood and self-reported health. Stress Medicine, 15, 189-196. http://dx.doi.org/10.1002/(SICI)1099-1700(199907)15: $3<189::$ AID-SMI814>3.0.CO;2-U

van Vliet, A. (2001). Homesickness: Antecedents, consequences and mediating processes. Utrecht, Germany: Utrecht University Press.

van Vliet, A. J., Stroebe, W., \& Schut, H. (1998). Verhuizen, heimwee en gezondheidsklachten bij eerstjaars studenten [Relocation, homesickness and health problems among Dutch university first year students]. Gedrag \& Gezondheid, 26, 91-100.

Vergara, M., Smith, N., \& Keele, B. (2010). Emotional intelligence, coping responses, and length of stay as correlates of acculturative stress among international university students in Thailand. Procedia: Social and Behavioral Sciences, 5, 1498-1504. http://dx.doi.org/10.1016/j.sbspro .2010.07.315

Verschuur, M., Eurelings-Bontekoe, E., \& Spinhoven, P. (2001). Construction and validation of the Homesickness Vulnerability Questionnaire. Personality and Individual Differences, 30, 11-19. http://dx.doi.org/ 10.1016/S0191-8869(00)00005-2

Verschuur, M., Eurelings-Bontekoe, E., Spinhoven, P., \& Duijsens, I. (2003). Homesickness, temperament and character. Personality and Individual Differences, 35, 757-770. http://dx.doi.org/10.1016/S0191$8869(02) 00281-7$

Vingerhoets, A. (2005). The homesickness concept: Questions and doubts. In M. van Tilburg \& A. Vingerhoets (Eds.), Psychological aspects of geographic moves: Homesickness and acculturation (pp. 1-15). Tilburg, the Netherlands: Tilburg University Press.

Ward, C., Bochner, S., \& Furnham, A. (2001). The psychology of culture shock (2nd ed.). Philadelphia, PA: Routledge.

Watt, S. E., \& Badger, A. J. (2009). Effects of social belonging on homesickness: An application of the belongingness hypothesis. Personality and Social Psychology Bulletin, 35, 516-530. http://dx.doi.org/ $10.1177 / 0146167208329695$

Ying, Y. (2005). Variation in acculturative stressors over time: A study of Taiwanese students in the United States. International Journal of Intercultural Relations, 29, 59-71. http://dx.doi.org/10.1016/j.ijintrel.2005 .04 .003 
Yu, B., Chen, X., Li, S., Liu, Y., Jacques-Tiura, A. J., \& Yan, H. (2014). Acculturative stress and influential factors among international students in China: A structural dynamic perspective. PLOS ONE, 9, e96322. http://dx.doi.org/10.1371/journal.pone.0096322

Zahra, D., Qureshi, A., Henley, W., Taylor, R., Quinn, C., Pooler, J., . . . Byng, R. (2014). The work and social adjustment scale: Reliability, sensitivity and value. International Journal of Psychiatry in Clinical
Practice, 18, 131-138. http://dx.doi.org/10.3109/13651501.2014 894072

Received November 24, 2014

Revision received March 11, 2015

Accepted March 12, 2015

\section{HISTORY OF PSYCHOLOGY}

CALL FOR PAPERS:

\section{DOES THE HISTORY OF PSYCHOLOGY HAVE A FUTURE?}

History of Psychology invites submissions for a special issue on the future of the history of psychology.

20 years ago, Kurt Danziger published an article with the provocative title, "Does the history of psychology have a future?" and it led to a great deal of comment and debate. The institutional position of the field does not seem to have improved in the meantime. The graduate program in history and theory of psychology at the University of New Hampshire was the only one of its kind in the USA and it was ended in 2009. Although the history of psychology is still widely taught at the undergraduate level, concerns have been expressed over a possible decline in the number of psychology departments offering the course. Professional historians have become increasingly prominent in the field. Could the subject eventually be handed over to them, as has already happened with the history of the physical sciences? Should this development be welcomed? There are many issues to be addressed.

We welcome contributions on any aspect of the subject. In order to get as many different perspectives as possible, we welcome contributions from authors in different disciplines (especially psychologists and historians), authors at different stages in their career (from graduate students to emeriti) and authors from different parts of the world. We are well aware that the current situation in the USA may not be representative of the situation elsewhere.

The submission deadline is July 15, 2015.

The main text of each manuscript, exclusive of figures, tables, references, or appendixes, should not exceed 35 double-spaced pages (approximately 7,500 words). Initial inquiries regarding the special issue may be sent to the editor, Nadine Weidman (weidman@fas.harvard.edu) or the guest editor, Adrian Brock (adrian.c.brock@gmail.com).

Papers should be submitted through the regular submission portal for History of Psychology (http://www.editorialmanager.com/hop/) with a cover letter indicating that the paper is to be considered for the special issue. Please see http://www.apa.org/pubs/journals/hop/ for Instructions to Authors. 